\title{
Regional Distribution of cGMP-Activated Ion Channels in the Plasma Membrane of the Rod Photoreceptor
}

\author{
Shu-Ichi Watanabe ${ }^{a}$ and Gary Matthews \\ Department of Neurobiology and Behavior, The State University of New York, Stony Brook, New York 11794
}

In patch-clamp recordings from excised membrane patches, the distribution of cGMP-activated channels in the plasma membrane of the rod photoreceptor was examined. These channels have been shown to be the light-sensitive channels that carry the inward dark current in the rod outer segment, where phototransduction occurs (Matthews, 1987); thus, they are centrally involved in photoreceptor transduction. In the outer segment, cGMP-activated channels were present at high density. In the inner segment, cGMP-activated channels were also present, but their density was much lower than in the outer segment. Calcium-activated potassium channels had the opposite distribution, with a higher density in the inner segment. The results suggest that there is a barrier preventing the unrestricted spread of membrane proteins between the inner and outer segments.

The rod photoreceptor of vertebrate retina can be functionally divided into the outer segment and the inner segment. The outer segment, where phototransduction occurs, is specialized for light reception, and its plasma membrane is thought to contain few if any ion channels other than the light-sensitive channels that carry the dark current (Baylor and Lamb, 1982; Baylor et al., 1984; Baylor and Nunn, 1986). In excised patches, these lightsensitive channels of the outer segment have been shown to be activated by guanosine $3^{\prime}: 5^{\prime}$-cyclic monophosphate (cGMP) (Matthews, 1987; Matthews and Watanabe, 1987). The inner segment, which is connected to the outer segment via a thin ciliary neck, contains a variety of voltage- and calcium-sensitive conductances (Bader et al., 1982) that shape the voltage response to illumination (Baylor et al., 1984). These inner segment conductances have not been observed in the outer segment. Thus, some sorting process apparently prevents inner scgment channels from spreading to the outer segment. In addition, rhodopsin immunoreactivity in the plasma membrane has been shown to be restricted to the outer segment (Spencer et al., 1986), which also implies a segregation mechanism operating on membrane proteins of inner and outer segments. To determine if this sorting process also restricts light-sensitive, cGMP-activated channels to the outer segment, inside-out membrane patches from rod inner segments were examined for cGMP-activated conductance. We found that cGMP-activated channels are present in the inner segment, but that their density is considerably lower than in the outer segment.

\footnotetext{
Received May 20, 1987; revised Nov. 9, 1987; accepted Nov. 11, 1987.

This work was supported by USPHS Grant EY03821.

Correspondence should be addressed to Dr. Matthews at the above address.

a Present address: Department of Physiology, Keio University School of Medicinc, 35 Shinano-machi, Shinjuku-ku, Tokyo 160, Japan.

Copyright (C) 1988 Society for Neuroscience $0270-6474 / 88 / 072334-04 \$ 02.00 / 0$
}

\section{Materials and Methods}

Patch-clamp recordings were made from outer and inner segments of single rod photoreceptors as described previously (Matthews, 1986; Matthews, 1987; Matthews and Watanabe, 1987). Rods were isolated from Bufo marinus retina by mechanical dissociation, with no enzyme treatment (Matthews, 1985). Mechanically isolated rods were typically intact except for the synaptic terminal, which apparently broke off during the dissociation. Some recordings were also made from rods with smaller, conically tapered inner segments, which evidently resulted when the cell was pinched off between the ellipsoid and the nuclcus.

Compositions of solutions are given in Table 1. Intact rods were bathed in normal Ringer's solution, and patch pipettes were filled with high $\mathrm{Na} / 0 \mathrm{Ca}$ solution. Solutions were applied to the intracellular face of excised patches using a modified version of the $Y$-tube perfusion system described in detail by Brett et al. (1986). In this system, the patch pirette is placed in the outflow leg of a Y-shaped tubing connector, and the solution flowing past the patch pipette is selected from 2 inlet tubes by a solenoid-activated, 2 -way pinch valve. cGMP $(2-1000 \mu \mathrm{M})$ was applied in high $\mathrm{Na} / 0.2 \mathrm{Ca}$ solution, and $\mathrm{Ca}$-activated $\mathrm{K}$ channels were examined by switching between high $\mathrm{K} / 0 \mathrm{Ca}$ and high $\mathrm{K} / 0.2 \mathrm{Ca}$ solutions.

\section{Results and Discussion}

cGMP-activated conductance in inner-segment patches

Of 62 excised patches obtained from rod inner segments, 22 $(35.5 \%)$ showed no detectable channel activity of any sort. These inactive patches may represent instances in which the ruptured ends of an excised patch sealed over to form a vesicle in the pipette tip. Therefore, we ignored all silent patches and focused attention on the remaining 40 patches in which some form of channel activity could be detected; such channel activity confirmed that an accessible patch, rather than a vesicle, had been formed. As summarized in Table 2, 25 of the 40 active patches $(62.5 \%)$ showed a conductance increase in response to application of cGMP to the intracellular membrane face. An example of the conductance increase produced by cGMP in an inner segment patch is shown in Figure $1 A$ (top trace). Because the conductance increase induced by cGMP in inner segment patches was typically small (see below), it was necessary to determine if the apparent cGMP response might be an artifact caused by slightly different flow rates in the 2 input lines of the Y-tube perfusion system. To examine such flow artifacts, the flow was switched between 2 identical solutions, both without cGMP. As shown in Figure $1 A$ (middle trace), there was no detectable conductance increase when both the normally flowing and the test solutions contained no cGMP; thus, the conductance increase in the presence of cGMP is attributable to cGMP-activated channels in the inner segment membrane. We found no detectable pattern in the regional distribution of cGMP-activated channels within the inner segment. We regularly obtained responsive patches from all parts of the inner segment: near the cilium connecting inner and outer segments, along the side of 


\begin{tabular}{|c|c|c|c|c|c|c|}
\hline Solution & $\mathrm{NaCl}$ & $\mathrm{KCl}$ & $\mathrm{CaCl}_{2}$ & $\mathrm{MgCl}_{2}$ & EDTA & $\begin{array}{l}\text { Glu- } \\
\text { cosc }\end{array}$ \\
\hline Normal Ringer & 111 & 2.5 & 1.0 & 1.6 & 0 & 10 \\
\hline $\mathrm{High} \mathrm{Na} / 0 \mathrm{Ca}$ & 117.5 & 0 & 0 & 0 & 0.15 & 0 \\
\hline $\mathrm{High} \mathrm{Na} / 0.2 \mathrm{Ca}$ & 117 & 0 & 0.2 & 0 & 0 & 0 \\
\hline High $\mathrm{K} / 0 \mathrm{Ca}$ & 4 & 113.5 & 0 & 0 & 0.15 & 0 \\
\hline $\mathrm{High} \mathrm{K} / 0.2 \mathrm{Ca}$ & 4 & 113 & 0.2 & 0 & 0 & 0 \\
\hline
\end{tabular}

Concentrations are given in $\mathrm{mm}$. All solutions contained $3 \mathrm{~mm}$ Tris and were adjusted to $\mathrm{pH}=7.8$ with $\mathrm{HCl}$.

the inner segment, and at the pole of the inner segment farthest from the connecting cilium, where the stalk leading to the synaptic pedicle would normally attach (mechanically dissociated rods almost always lack a synaptic terminal). Also, among responsive inner-segment patches, there was no apparent regional pattern within the inner segment in the maximal amplitude of the cGMP-activated conductance, so that channel density did not seem to vary in a consistent way in different parts of the inner segment.

\section{Calcium-activated $K$ conductance in inner-segment patches}

Fifteen of 40 inner-segment patches lacked cGMP-activated conductance, although other channels were present. As described above, the presence of these other channels confirmed that a vesicle had not formed. The most common other type of conductance encountered in inner-segment patches was calcium-activated $\mathrm{K}$ conductance of $[\mathrm{K}(\mathrm{Ca})]$, which was present in $45 \%$ of the patches (see Table 2 ). An example of an innersegment patch with $\mathrm{K}(\mathrm{Ca})$ but without cGMP-activated conductance is shown in Figure $1 B$. To test for the presence of $\mathrm{K}(\mathrm{Ca})$ in an excised patch, the membrane potential was clamped to $0 \mathrm{mV}$, and the intracellular face of the membrane was superfused with a high-potassium solution (Table 1). Under thesc conditions, only potassium could carry an outward membrane current. The intracellular solution was then switched from 0 to $0.2 \mathrm{~mm} \mathrm{Ca}$, at constant high $\mathrm{K}$, to activate $\mathrm{K}(\mathrm{Ca})$. As illustrated in the middle trace of Figure $1 B$, application of high $\mathrm{K} / 0.2 \mathrm{Ca}$ solution induced a large outward current. This established the presence of calcium-activated $\mathrm{K}$ conductance and was the usual means of establishing that a patch lacking cGMP-activated conductance was not a vesicle. Other types of non-calcium-activated channels were found in 8/40 inner-segment patches, but these were not characterized further.

\section{Maximal amplitude of $C G M P$-activated conductance in outer- and inner-segment patches}

For comparison with inner-segment patches, excised patches were obtained from outer segments of rods in the same preparations. In this series of experiments, $100 \%$ of outer-segment patches responded to intracellular application of cGMP (Figure 1C). Thus, cGMP-activated conductance was present more frequently in outer-segment than in inner-segment patches, where it was found in $62.5 \%$ of patches. In addition, the maximum amplitude of the cGMP-activated conductance at a saturating dose (0.1-1.0 mM) of cGMP was typically much larger in outer segment patches. In 40 inner-segment patches, maximal cGMPactivated conductance averaged $46.5 \pm 23.8 \mathrm{pS}$ (mean \pm SEM), while in 70 outer segment patches the average was $665 \pm 106$ $\mathrm{pS}$ (mean $\pm \mathrm{SEM}$ ). The distributions of maximal conductance
A
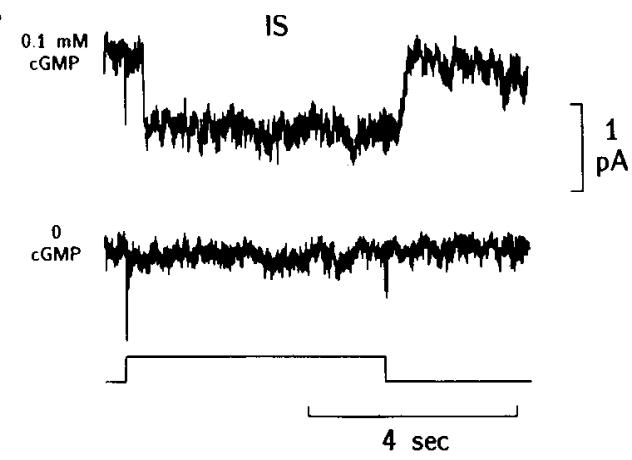

B
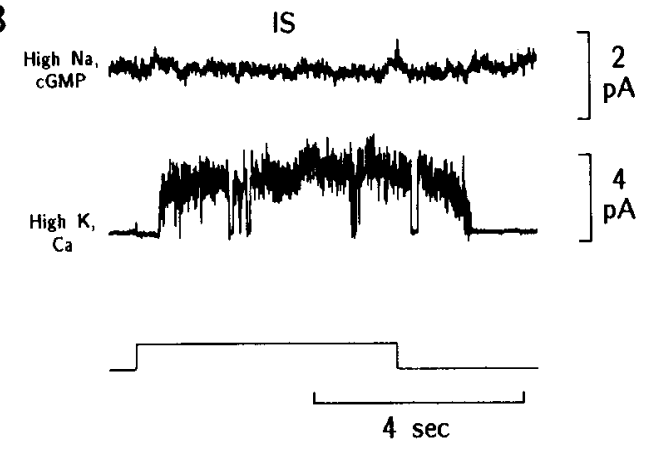

C

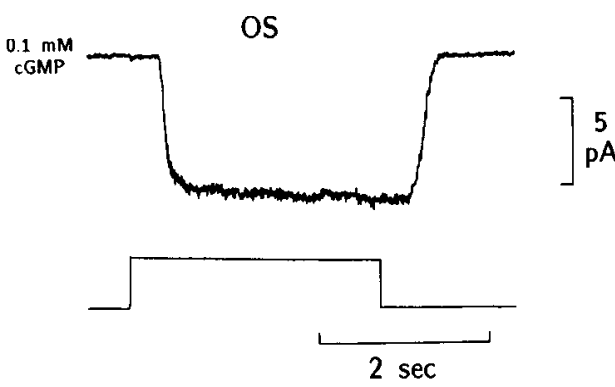

Figure 1. cGMP-activated conductance in excised inner- and outersegment patches. $A-C$, Bottom traces show the pulse controlling the solenoid-activated valve that selected the solution flowing past the patch pipette. In each case, there was a delay between the beginning of the switching pulse and the onset of the conductance change. This is largely due to propagation time from the inlet port to the patch within the outflow leg of the Y-tube. $A$, Conductance change induced by cGMP applied to intracellular face of an inside-out inner-segment patch (IS). Upper trace, Intracellular solution switched from high Na/0.2 Ca (Table 1) to the same solution with $0.1 \mathrm{~mm}$ cGMP. At the patch membrane potential of $-39 \mathrm{mV}$, cGMP induced an inward membrane current. To control for flow artifacts (middle trace), the experiment was repeated with both inlet solutions having no cGMP. Traces are averages of 6 (upper) or 7 (middle) successive presentations. Recording bandwidth, $0-100 \mathrm{~Hz}, B$, An inside-out inner-segment patch that did not respond to cGMP, although calcium-activated $\mathrm{K}$ conductance was present. $U p$ per trace, the intracellular solution was switched from high $\mathrm{Na} / 0.2 \mathrm{Ca}$ to the same solution with $0.1 \mathrm{mM}$ cGMP; there was no cGMP-induced conductance change (patch membrane potential, $-41 \mathrm{mV}$ ). Middle trace, the intracellular solution was switched from high $\mathrm{K} / 0 \mathrm{Ca}$ (Table 1) to high $\mathrm{K} / 0.2 \mathrm{Ca}$, activating an outward membrane current carried by potassium. Patch membrane potential, $0 \mathrm{mV}$. Upper trace is an average of 4 sweeps, while the middle trace is a single sweep. Bandwidth, 0-100 Hz. $C$, cGMP-activated conductance in an inside-out outer-segment patch $(O S)$. The intracellular solution was switched from high $\mathrm{Na} / 0.2$ Ca solution to the same solution with $0.1 \mathrm{~mm}$ cGMP. The upper trace is the average of 5 presentations. Patch membrane potential, $-21 \mathrm{mV}$; bandwidth, $0-80 \mathrm{~Hz}$. 
Table 2. Summary of different types of ion channel found in insideout patches obtained from inner segments and outer segments of rod photoreceptors

\begin{tabular}{|c|c|c|}
\hline Type of channel & $\begin{array}{l}\text { Inner segment } \\
(\%)\end{array}$ & $\begin{array}{l}\text { Outer segmen } \\
(\%)\end{array}$ \\
\hline No activity & $22 / 62(35.5)$ & $0 / 25(0)$ \\
\hline Only cGMP-activated & $14 / 40(35.0)$ & $22 / 25(88.0)$ \\
\hline cGMP-activated $+\mathrm{K}(\mathrm{Ca})$ & $8 / 40(20.0)$ & $3 / 25(12.0)$ \\
\hline cGMP-activated + other channel ${ }^{a}$ & $3 / 40(7.5)$ & $0 / 25(0)$ \\
\hline OnlyK(Ca) & $10 / 40(25.0)$ & $0 / 25(0)$ \\
\hline Only other channel ${ }^{a}$ & $5 / 40(12.5)$ & $0 / 25(0)$ \\
\hline Total cGMP-activated & $25 / 40(62.5)$ & $25 / 25(100)$ \\
\hline Total K(Ca) & $18 / 40(45.0)$ & $3 / 25(12.0)$ \\
\hline
\end{tabular}

Figures for outer-segment patches include only the 25 patches that were specifically tested for the presence of calcium-activated potassium channels [K(Ca)].

a "Other channels" include non-calcium-activated $\mathrm{K}$ channels and channels of unknown type (not $\mathrm{K}$ channels).

for all inner- and outer-segment patches are shown in Figure 2, which shows that there is little ovcrlap of the 2 distributions. The difference in maximal conductance shown in Figure 2 is unlikely to be the result of differences in the area of the excised patches from inner and outer segments. Pipettes were constructed in the same way, and excised patches were formed in the same manner in both cases. Also, a lower limit on patch area is set by the area of the pipette lumen (about $0.2 \mu \mathrm{m}^{2}$ ). If the outer-segment patches were larger than inner-segment patches by an amount sufficient to account for the difference in maximal cGMP-activated conductance, it would require that a minimum of $3 \mu \mathrm{m}^{2}$ of outer segment membrane enters the patch pipette at the recording site, producing a readily visible constriction of the outer segment. No such constriction was observed, and no material could be seen to enter the patch pipette in either inneror outer-segment recordings. Therefore, patch areas are likely similar, and the difference in maximal conductance indicates a difference in channel density.

\section{Comparison of cGMP-activated channels in inner- and outer- segment patches}

In both inner- and outer-segment patches, the cGMP-activated conductance was saturated by $0.1-0.5 \mathrm{~mm}$ cGMP. When the concentration of CGMP was lowered to 5-20 $\mu \mathrm{M}$, individual opcnings of single cGMP-activated channels could be observed in inner-segment patches, as in outer-segment recordings. The single-channel conductance averaged $25.6+0.9 \mathrm{pS}$ (mean \pm $\mathrm{SEM}, n=11$ ) in inner-segment patches, which is comparable to the conductance of single cGMP-activated channels in outersegment patches $(24.0 \pm 1.2 \mathrm{pS}$; Matthews and Watanabe, 1987). To compare the kinetics of channel events in inner and outer segments, we examined the noise properties of the cGMP-activated current in inner-segment patches, as done previously for the cGMP-activated conductance in outer-segment patches (Matthews, 1986). As in the outer-segment recordings, the power spectrum of cGMP-activated channel activity in the inner segment was fitted by a sum of 2 Lorentzian components, with average corner frequencies of $62 \pm 9$ and $868 \pm 106 \mathrm{~Hz}$ (mean \pm SEM, $n=6$ ) for the low- and high-frequency components. These are similar to the corner frequencies of 70 and $1190 \mathrm{~Hz}$ reported by Matthews (1986) for the cGMP-activated conductance in the outer segment. Thus, cGMP-activated channels like those of the
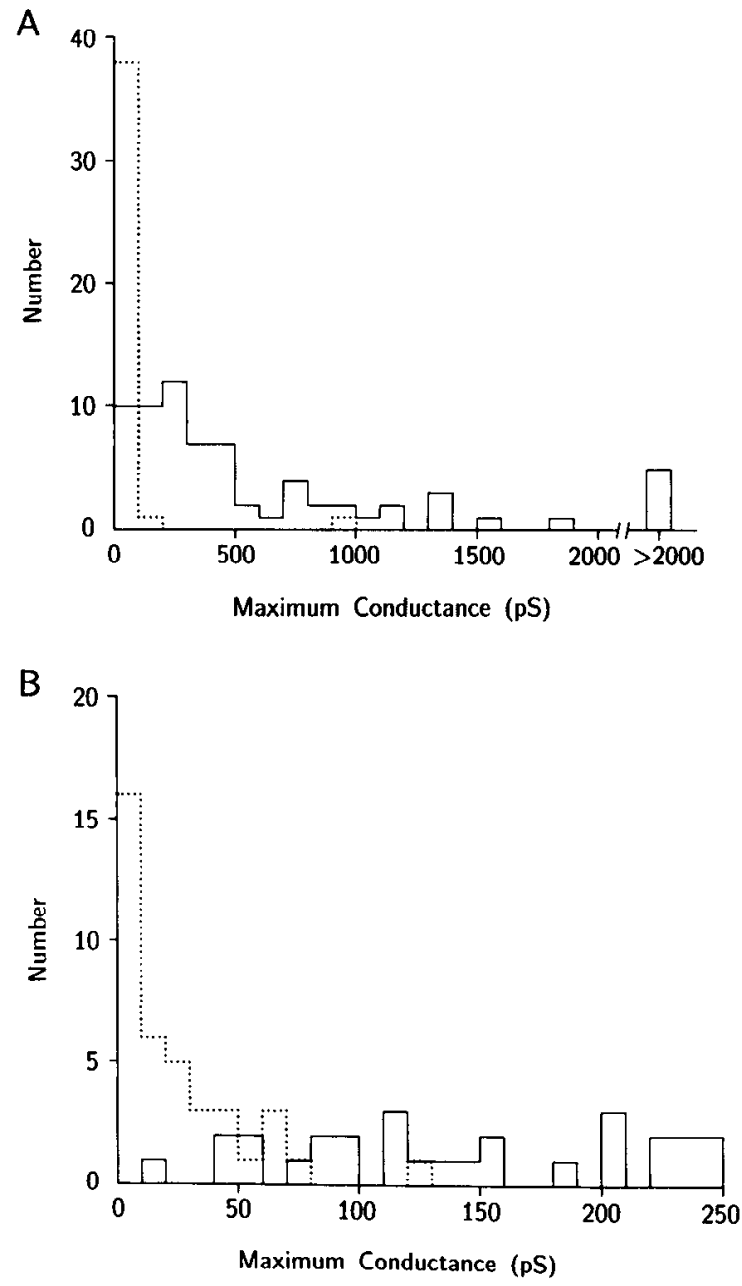

Figure 2. Distribution of maximal cGMP-activated conductance for inner-segment (dotted line) and outcr-scgmcnt (solid line) patches. $A$, Full distributions. $B$, Expanded view of distributions at maximal conductances less than $250 \mathrm{pS}$. All conductances were measured in the steady state at a recording bandwidth of $0-80 \mathrm{~Hz}$. Maximal conductances less than $100 \mathrm{pS}$ were measured from averages of from 4 to 17 individual responses.

outer segment are present in the plasma membrane of the inner segment, but the channel density in the inner segment is considerably lower than in the outer segment. This lower channel density in the inner segment is reflected both in the lower percentage of inner-segment patches containing cGMP-activated channels and in the smaller maximum conductance increase in those inner-segment patches that did respond to cGMP.

\section{Calcium-activated $K$ conductance in outer-segment patches}

For the sake of symmetry in Table 2, we also tested for the presence of $\mathrm{K}(\mathrm{Ca})$ in 25 outer-segment patches, as described above for inner-segment patches. In 3 of those patches (12.0\%), $\mathrm{K}(\mathrm{Ca})$ channels were observed (Table 2). A recording of $\mathrm{K}(\mathrm{Ca})$ activity in an outer-segment patch, together with similar activity in an inner-segment patch for comparison, is shown in Figure 3. We have not examined these channels in detail because we recorded their activity in the inner segment merely as a means of establishing that the intracellular face of an excised patch was accessible and that a vesicle had not formed. However, we mention the cxistence of these channels in the outer segment because their presence there was surprising, given previous reports that 


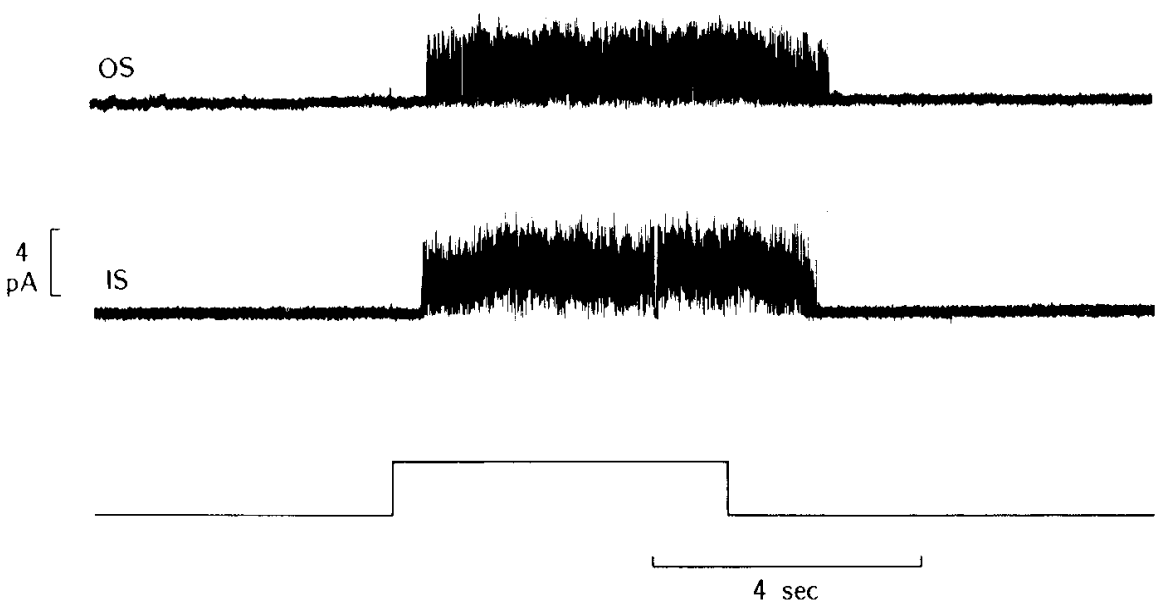

Figure 3. Calcium-activated potassium conductance in outer-segment (top) and inner-segment (middle) patches. The pipette solution was high $\mathrm{Na} / 0 \mathrm{Ca}$, and the solution bathing the intracellular face of the membrane contained high potassium. At the patch membrane potential of $0 \mathrm{mV}$, only potassium ions could carry outward current. During the upward deflection in the bottom trace, the intracellular solution was switched from high $\mathrm{K} / 0 \mathrm{Ca}$ to high $\mathrm{K} / 0.2 \mathrm{Ca}$ to activate calcium-activated potassium channels. Bandwidth, 0-320 $\mathrm{Hz}$; note the difference in bandwidth from Figure $1 B$.

the outer segment contains only light-sensitive channels (Baylor and Lamb, 1982; Baylor et al., 1984; Baylor and Nunn, 1986). The current through these $\mathrm{K}(\mathrm{Ca})$ channels is sufficiently large (see Fig. 3) that the opening of even one channel should be apparent in either suction-electrode (Baylor and Lamb, 1982; Baylor et al., 1984) or voltage-clamp (Gray and Attwell, 1985; Baylor and Nunn, 1986) recordings of outer segment membrane current in intact rods, but such openings have not been reported. Also, we have never seen outward-current channels in hundreds of cell-attached patch-clamp recordings from outer segments of dark-adapted rods. Thus, $\mathrm{K}(\mathrm{Ca})$ channels in the outer segment apparently do not open under physiological conditions. This in turn suggests that internal calcium in the outer segment of the intact rod is too low to activate $\mathrm{K}(\mathrm{Ca})$ channels in either darkness or light or that these channels are somehow masked in the intact rod.

\section{Conclusion}

Some sorting process apparently acts to segregate channels to the inner and outer segments. Exclusion of inner-segment ion channels from the outer segment may have functional significance: because the outer segment is a long cylinder, small photocurrents will propagate more efficiently to the inner segment if there are few ion channels other than the light-sensitive channels themselves to provide a leakage pathway. However, the opcration of the segregation mechanism is not perfect; some calcium-activated potassium channels were found in the outer segment, and some cGMP-activated channels were found in the inner segment. As argued above, $\mathrm{K}(\mathrm{Ca})$ channels in the outer segment probably have no physiological function. We do not know, however, whether cGMP-activated channels in the inner segment play some physiological role or are merely an incidental result of the imperfect operation of the channel sorting process.

Note added in proof: Hestrin and Korenbrot (1987) have recently reported noncalcium-activated, voltage-dependent potassium channels in rod outer segments when rods were isolated by enzymatic treatment, but not when rods were isolated by mechanical means. They concluded that enzymatic dissociation was somehow responsible for the presence of the potassium channels in the outer segment. However, in our experiments, no enzyme treatment was used yet we found calcium-activated potassium channels in outer-segment patches.

\section{References}

Bader, C. R., D. Bertrand, and E. A. Schwartz (1982) Voltage-activated and calcium-activated currents studied in solitary rod inner segments from the salamander retina. J. Physiol. (Lond.) 331: 253-284.

Baylor, D. A., and T. D. I amb (1982) Local effects of bleaching in retinal rods of the toad. J. Physiol. (Lond.) 328: 49-71.

Baylor, D. A., and B. J. Nunn (1986) Electrical properties of the lightsensitive conductance of rods of the salamander Ambystoma tigrinum. J. Physiol. (Lond.) 371: 115-145.

Baylor, D. A., G. Matthews, and B. J. Nunn (1984) Location and function of voltage-sensitive conductances in retinal rods of the salamander Ambystoma tigrinum. J. Physiol. (Lond.) 354: 203-223.

Brett, R. S., J. P. Dilger, P. R. Adams, and B. Lancaster (1986) A method for the rapid exchange of solutions bathing excised membrane patches. Biophys. J. 50: 987-992.

Gray, P., and D. Attwell (1985) Kinetics of light-sensitive channels in vertebrate photoreceptors. Proc. R. Soc. Lond. [Biol.] 223: 379388 .

Hestrin, S., and J. I. Korenbrot (1987) Voltage-activated potassium channels in the plasma membrane of rod outer segments: A possible effect of enzymatic cell dissociation. J. Neurosci. 7: 3072-3080.

Matthews, G. (1985) Membrane current noise in toad retinal rods exposed to low external calcium. J. Physiol. (Lond.) 361: 609-617.

Matthews, G. (1986) Comparison of the light-sensitive and cyclic GMP-sensitive conductances of the rod photoreceptor: Noise characteristics. J. Neurosci. 6: 2521-2526.

Matthews, G. (1987) Single-channel recordings demonstrate that cGMP opens the light-sensitive ion channel of the rod photoreceptor. Proc. Natl. Acad. Sci. USA 84: 299-302.

Matthews, G., and S.-I. Watanabe (1987) Properties of ion channels closed by light and opened by guanosine $3^{\prime}, 5^{\prime}$-cyclic monophosphate in toad retinal rods. J. Physiol. (Lond.) 389: 691-715.

Spencer, M., P. B. Detwiler, and A. H. Bunt-Milam (1986) Opsin redistribution in rods with fused inner and outer segments. Invest. Ophthalmol. Vis. Sci. 27: 235. 